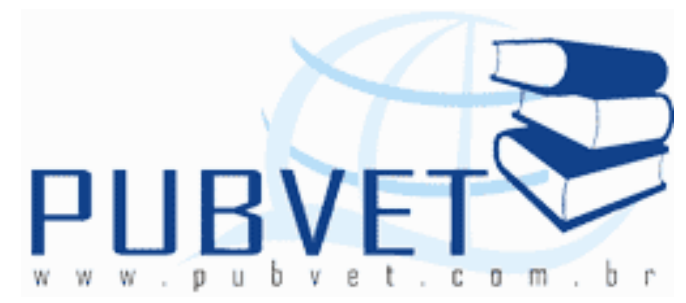

PUBVET, Publicações em Medicina Veterinária e Zootecnia.

https://doi.org/10.31533/pubvet.v02n12a466.1-8

\title{
Sinopse preliminar sobre a ocorrência de siris nas regiões estuarinas do Estado do Ceará
}

\section{Marco Antonio Igarashi}

Professor do Departamento de Engenharia de Pesca da UFC a disposição do SEAP/PR

\section{Resumo}

O ambiente estuarino é um importante e favorável local para reprodução e desenvolvimento de um grande número de siris. Várias espécies de siris ocupam a mesma área ecológica, incluindo áreas de manguezais. No Estado do Ceará, Região Nordeste do Brasil, estes animais participaram com um pequeno volume no mercado consumidor, sendo capturados por meio da pesca artesanal. O objetivo do artigo será de auxiliar no conhecimento de determinados tipos de siri que está sendo manuseado. Os seguintes siris foram identificados: Callinectes affinis (Fausto-Filho, 1980) C. bocourti (Milne Edwards, 1879), C. danae (Smith, 1869), C. ornatus (Ordway, 1863), C. exasperatus (Gerstaecker, 1856), C. larvatus (Ordway, 1863), C. sapidus (Rathbun, 1896) e Portunus spinimanus (Latreille, 1819). Esta pesquisa enfatiza a importância em desenvolver os mangues no sentido de suportar a captura de seus recursos pesqueiro tais como os siris e melhorar a economia costeira.

Palavras Chave: ocorrência - siris - estuárinas 


\title{
Preliminary study occurrence of swimming crabs in estuarine regions of Ceara state
}

\begin{abstract}
Estuarine ambient is a favorable and important site for the reproduction and growth of a large number of swimming crabs. Various species of swimming crabs occupy much the same ecological area, including mangrove areas. In Ceará State, Northeastern region of Brazil, swimming crabs have a small market volume and crab fishing is artisanal. This will help you to be considerable more knowledgeable in determining which types of swimming crabs you are handling. The following swimming crabs were identified: Family Portunidae: Callinectes affinis (Fausto-Filho, 1980), C. bocourti (Milne Edwards, 1879), C. danae (Smith, 1869), C. ornatus (Ordway, 1863), C. exasperatus (Gerstaecker, 1856), C. larvatus (Ordway, 1863), C. sapidus (Rathbun, 1896) and Portunus spinimanus (Latreille, 1819). This research emphasizes the importance of developing more mangroves for supporting the swimming crab fishery resources and enhancing the coastal economies.
\end{abstract}

Key Words: occurrence - swimming crabs - estuarine

\section{Introdução}

Os crustáceos decápodos são encontrados em abundância nos ambientes estuarinos do Estado do Ceará, Brasil e, dentre estes dependendo da espécie, os siris.

Quanto a ocorrência de espécies de crustáceos no Estado do Ceará, poucos trabalhos foram realizado em relação as espécies de siris (CAMPOS, 1995, PARENTE, 1984).

Embora provavelmente não conste das estatísticas oficiais (IPLANCE, IBAMA) dados econômicos sobre a captura de siris nas zonas estuarinas do 
Estado do Ceará, constatou-se, através de levantamentos faunístico nestas áreas, uma diminuição populacional de algumas espécies de siris. Desta forma este trabalho, tem como objetivo ordenar as informações sobre os siris que podem ocorrer nos estuários do Estado do Ceará, como forma de contribuir para o conhecimento de sua diversificação.

\section{Espécies de siri}

Devido a grande quantidade de alimentos, o ambiente estuarino possui grande fertilidade natural. Neste contexto fazendo parte deste ambiente têmse os manguezais, responsáveis pela produção orgânica. Têm função primordial no abastecimento de detritos vegetais no substrato, importantes para os siris, que podem utilizar estes detritos como alimento. Portanto os estuários com o mangue são habitados por uma fauna bastante rica; dentre os organismos bentônicos, estão os siris.

Os siris têm grande importância na composição faunística do ambiente estuarino, tanto por sua abundância e participação na cadeia alimentar, como por outras funções ecológicas que desempenham. Todavia os siris conseguem suportar variações da quantidade de sal na água, e por isso encontram-se com maior freqüência nas zonas do manguezal próximas do mar.

Quanto aos valores de salinidade, se registrou uma forte variação que depende das estações de coleta, sendo em geral registradas as salinidades mais baixas que coincide com os meses de chuvas, durante os meses de alta salinidade coincide similarmente com o período de secas na região.

Os portunídeos distribuem-se ao longo da costa Atlântica Ocidental, desde a América do Norte até o extremo sul da América do Sul (FAO, Melo, citado por BAPTISTA et al., 2003).

No Brasil são registradas 21 espécies de siris da família Portunidae (MELO, 1996; MANTELATTO \& DIAS, 1999 citado por FERNANDES et al., 2006). 
As espécies do gênero Callinectes nativas ou introduzidas se encontram nas costas europeias (WILLIAMS, 1965), Japão (LEMAITRE, 1981), região oriental do Mediterrâneo (CHACE \& HOBBS, 1969), África ocidental e ocasionalmente no Indopacífico; na América espécies nativas do gênero se encontram em ambas as costas e hemisférios (RODRÍGUEZ, 1980).

Várias especies alcançam a região em estudo: Callinectes larvatus (que se distribui desde o Norte da Carolina até o Paraná), C. bocourti e $C$. exasperatus (Florida-Santa Catarina), C. danae (Florida-Uruguai) (SPIVAK, 1997).

Coelho e Ramos-Porto citado por MENDES et al. (1997) citam seis espécies de siris que ocorrem no Brasil, o C. danae, C. bocourti, C. larvatus, $C$. exasperatus, $C$. sapidus e o $C$. ornatus.

Por outro lado no presente estudo entre as espécies identificadas está a C. affinis (Fausto-Filho, 1980), encontrado nos complexos estuarinos dos rios Cocó e Ceará, nos arredores da região metropolitana de Fortaleza, em fundos de lama e regiões estuarinas; suporta uma variação de salinidade de 0 a 35 $\%$, parece ser bastante tolerante a águas poluídas e estagnadas (CAMPOS, 1995).

Tabela 1. Espécies de siris que podem ser encontrados nos estuários do Estado do Ceará

\begin{tabular}{ccc}
\hline Número & Espécies \\
\hline 1 & Callinectes affinis (Fausto-Filho, 1980) & Portunidae \\
2 & Callinectes bocourti (Milne Edwards, 1879) & $"$ \\
3 & Callinectes danae (Smith, 1869) & $"$ \\
4 & Callinectes ornatus (Ordway, 1863) & $"$ \\
5 & Callinectes exasperatus (Gerstaecker, 1856) & $"$ \\
6 & Callinectes larvatus (Ordway, 1863) & $"$ \\
7 & Callinectes sapidus (Rathbun, 1896) & "
\end{tabular}


Na Familia Portunidae os siris Callinectes spp. são abundantes em águas estuarinas e costeiras de regiões temperadas e tropicais; os adultos, em geral, se alimentam de invertebrados e pequenos peixes. No Estado do Ceará, os portunídeos que se têm destacado na presente pesquisa por sua abundância são C. bocourti e C. danae.

Callinectes affinis (Fausto-Filho, 1980)

Podem ser encontrados nas regiões estuarinas dos rios Cocó e Ceará; portanto vivem em regiões estuarinas em fundos de lama, suportando variação de salinidades de 0 a 35\%o, sugerindo que esta espécie tolera as águas poluídas e estagnadas, principalmente com as observações realizadas nestes rios próximos a região metropolitana de Fortaleza (CAMPOS, 1995). Contudo esta espécie foi uma das mais encontradas do gênero nos manguenzais circunvizinhas a Fortaleza. No Estado do Ceará, a comercialização de $C$. affinis vem sendo feita principalmente pela população ribeirinha de baixa renda e por pescadores que fazem da pesca diária de siri seu meio de subsistência e a base da alimentação de suas famílias; podem ser capturados com redes de arrasto de fundo e puçás com iscas (CAMPOS, 1995). Pode ser encontrada a venda viva amarrada a corda ao longo das rodovias.

Callinectes bocourti (Milne Edwards, 1879)

Nome vulgar: siri (Fausto-Filho, 1966), siri pimenta (CAMPOS, 1995). Podem ser encontradas nos estuários próximos a Fortaleza e redondeza ou regiões costeiras de pouca profundidade, vive em fundos de lama, areia, rochoso e de fragmentos de conchas. Sugere-se que se alimenta de invertebrados, peixes e detritos. Pode ser capturado com redes de arrasto de fundo e puçás iscados. Pode ser encontrada a venda em cordas ao longo das rodovias. No Estado do Ceará, a comercialização de $C$. bocourti vem também sendo feita principalmente pela população ribeirinha de baixa renda. A captura de siris nos estuários e áreas litorâneas do Estado do Ceará pode ser uma alternativa de 
subsistência para pescadores artesanais. Portanto esta espécie apresenta interesse sócio-econômico.

Callinectes danae (Smith, 1869)

$\mathrm{Na}$ costa do Estado do Ceará esta espécie, vive em fundos de lama e manguezais em regiões estuarinas, até fundos de fragmentos de conchas recobertos de algas, estendendo - se desde as praias até o mar aberto em profundidades de $75 \mathrm{~m}$ (CAMPOS, 1995). Tolera grandes variações de salinidades e provavelmente águas hipersalinas. Podendo ser comercializado ao longo das rodovias em mercados locais e regionais. No entanto, em termos de captura só existe registro oficial do $C$. danae, com um volume de extração acumulado de 52.104 toneladas, entre 1984 à 1995 e com esta estatística, o Brasil posicionou-se como o terceiro maior país a capturar esse crustáceo (FAO,1997).

Callinectes ornatus (Ordway, 1863)

Nas proximidades de Fortaleza é uma espécie bastante capturada, normalmente com rede de arrasto e tolera uma ampla faixa de salinidade (de 0 a $50 \%$ ) e alimenta-se presumivelmente de uma ampla variedade de materiais, desde moluscos e outros invertebrados bentônicos até alguns peixes e detritos.

Callinectes exasperatus (Gerstaecker, 1856)

Habita regiões estuarinas, especialmente em associação com manguezais nas desembocaduras de rios, alimenta-se provavelmente de uma grande variedade de materias desde molusco e outros invertebrados bentônicos até a alguns peixes e detritos (CAMPOS, 1995). Conhecido como "siriaçu", não é muito abundante, apresenta pequeno porte, e pouco valor comercial (PARENTE, 1984). 
Callinectes larvatus (Ordway, 1863)

Nome vulgar siri-azul, espécie pouco abundante, pode ser encontrado em uma variedade de ambientes de águas rasas. Supõe-se que é uma espécie de pouco valor comercial.

Callinectes sapidus (Rathbun, 1896).

Pouco abundante, capturado ocasionalmente, toleram grandes variações de salinidade (0 a 48 \%) (CAMPOS, 1995).

Portunus spinimanus (Latreille, 1819)

Nome vulgar: siri-canela, encontrado em fundos de areia e lama próximo a desembocadura de rios como o Rio Ceará em Fortaleza (CAMPOS, 1995)

\section{Considerações finais}

A chave de ação é vencer desafios e encontrar meios para criação de condições sob as quais as pessoas possam explorar o siri sem afetar o meio ambiente. É mister a criação de novas leis, a níveis nacional e internacional para regular a relação entre pescadores no sentido de se proteger o ambiente natural e promover desenvolvimento de exploração sustentável.

Os siris analisados são importantes para o ecossistema estuarino, fazendo parte da cadeia trófica assim como algumas espécies são comercialmente e socialmente importantes servindo de sustento e fonte de renda para inúmeras famílias que vivem de sua captura. No Estado do Ceará a comercialização de siris vem sendo feita principalmente pela população de baixa renda e por pescadores que fazem da pesca diária de siri seu meio de subsistência e a base da alimentação de suas famílias.

\section{Referências bibliográficas}

BAPTISTA, C.; PINHEIRO, M. A. A.; BLANKENSTEYN, A ; BORZONEL, C. A. 2003. Estrutura populacional de Callinectes ornatus Ordway (Crustacea, Portunidae) no Balneário Shangri- 
Igarashi, M.A. Sinopse preliminar sobre a ocorrência de siris nas regiões estuarinas do Estado do Ceará. PUBVET, Londrina, V. 2, N. 49, Art\#466, Dez2, 2008.

Lá, Pontal do Paraná, Paraná, Brasil. Disponível em: http://www.scielo.br/ scielo.php?script $=$ sci arttext\&pid $=$ S0101-81752003000400018\&lng $=$ en\&nrm $=$ iso\&tlng $=p t$ Rev. Bras. Zool. vol.20 no.4 Curitiba Dec. 2003 (acesso 8.6.2004)

CAMPOS, A. A. 1995. Crustáceos decápodos do Nordeste brasileiro - Lista sistemática e guia de identificação das espécies de interesse comercial. DISSERTAÇÃO APRESENTADA AO DEPARTAMENTO DE ENGENHARIA DE PESCA DOCCA DA UFC COMO PARTE DAS EXIGÊNCIAS PARA OBTENÇÃO DO TÍTULO DE ENGENHEIRO DE PESCA. Fortaleza-CE, 173 p.

CHACE Jr., F. A.; HOOBS, H. H. 1969. The Freshwater and Terrestrial Decapod Crustaceans of the West Indies with Special Reference to Dominica. U.S. Nat. Mus. 292. 258 p.

FAO. AQUACULT-PC. 1997. Fishery information, data and statistics (FIDI), time series of production from aquaculture (quantities and values) and capture fisheries (quantities). Programa computacional.

FERNANDES, J. M.; ROSA, D. M.; ARAUJO, C. C. V.; RIPOLI, L. V. \& SANTOS, H. S. 2006. iologia e distribuição temporal de Callinectes ornatus Ordway, 1863 (Crustacea, Portunidae) em uma praia arenosa da Ilha do Frade, Vitória-ES. BOL. MUS. BIOL. MELLO LEITÃO (N. SÉR.), 20:59-71.

LEMAITRE, R., B., Jr. 1981. Shallow-water crabs (Decapoda, Brachyura). Collected in the Southern Caribbean Near Cartagena, Mar. Sci., Colombia. 31 (2): 234-266.

MANTELATTO, F. L. M. \& DIAS, L. L. 1999. Extension of the know distribuition of Charybdis hellerii (A. Milne- Edwards, 1867) (Decapoda, Portunidae) along the western tropical South Atlantic. Crustaceana, 72 (6): 617-620.

MELO, G. A. S. 1996. Manual de identificação dos brachyura (caranguejo e siri) do litoral brasileiro. Plêaide/Edusp, São Paulo, 604p.

MENDES, P. P.; CAVALLAZZI, F. E.; VIANA, G. F. S. 1997. Morfometria em siris, do gênero Callinectes stimpsom, 1860 (crustacea, decapoda, brachyura), nos rios serrambi, jaboatão e timbó do estado de Pernambuco. CONGRESSO BRASILEIRO DE ENGENHARIA DE PESCA GUARAPARI, ES. 3 a 6 de novembro de 1997. Anais publicado em CDRoom. p. 559-564.

PARENTE, P. M. 1984. generalidades sobre a biologia, ecologia e sistemática dos sisris do gênero Callinestes (Stimpson, 1860) no litoral do Estado do Ceará (Crustácea, Decapoda, Prtunidae).Dissertação apresentada ao Departamento de Engenharia de Pesca doCCA da UFC como parte das exigências para obtenção do título de Engenheiro de Pesca. FortalezaCE, junho de 1984. $31 \mathrm{p}$.

RODRíGUEZ, G. 1980. Los Crustáceos Decápodos de Venezuela. Inst. Venezolano de Investig. Científicas. Caracas, Venezuela, 494 p.

WILLIAMS, A.B. 1965. Marine Decapod Crustaceans of the Carolina. Fish Bull. 65 (1): 298 p.

SPIVAK, E. D. 1997. Cangrejos estuariales del Atlántico sudoccidental (250-41오) (Crustacea: Decapoda: Brachyura). Invest. Mar. Valparaíso, 25: 105-120. Disponível em: http://www.scielo.cl/ scielo. php? script=sci _arttext\&pid=S0717$71781997002500008 \& \mathrm{lng}=\mathrm{en} \& \mathrm{nrm}=\mathrm{iso} \& \mathrm{t} \operatorname{lng}=\mathrm{es}$ (acesso 10.06.2004) 